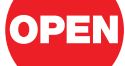

SUBJECT AREAS:

SURFACE PATTERNING

PHOTONIC CRYSTALS

LIQUID CRYSTALS

NANOPARTICLES

Received

20 August 2012

Accepted

29 November 2012

Published

28 February 2013

Correspondence and requests for materials should be addressed to

G.U.K. (kulkarni@ incasr.ac.in) or M.S.M.S. (saifullahm@ imre.a-star.edu.sg)

\section{Metal hierarchical patterning by direct nanoimprint lithography}

\author{
Boya Radha ${ }^{1,2}$, Su Hui Lim ${ }^{2,3}$, Mohammad S. M. Saifullah² \& Giridhar U. Kulkarni'
}

${ }^{1}$ Chemistry and Physics of Materials Unit and DST Unit on Nanoscience, Jawaharlal Nehru Centre for Advanced Scientific Research, Jakkur P.O., Bangalore 560064 , India, ${ }^{2}$ Institute of Materials Research and Engineering, A*STAR (Agency for Science, Technology and Research), 3 Research Link, Singapore 1 17602, Republic of Singapore, ${ }^{3}$ Department of Electrical and Computer Engineering, National University of Singapore, 4 Engineering Drive 3, Singapore 117576, Republic of Singapore.

Three-dimensional hierarchical patterning of metals is of paramount importance in diverse fields involving photonics, controlling surface wettability and wearable electronics. Conventionally, this type of structuring is tedious and usually involves layer-by-layer lithographic patterning. Here, we describe a simple process of direct nanoimprint lithography using palladium benzylthiolate, a versatile metal-organic ink, which not only leads to the formation of hierarchical patterns but also is amenable to layer-by-layer stacking of the metal over large areas. The key to achieving such multi-faceted patterning is hysteretic melting of ink, enabling its shaping. It undergoes transformation to metallic palladium under gentle thermal conditions without affecting the integrity of the hierarchical patterns on micro- as well as nanoscale. A metallic rice leaf structure showing anisotropic wetting behavior and woodpile-like structures were thus fabricated.

Furthermore, this method is extendable for transferring imprinted structures to a flexible substrate to make them robust enough to sustain numerous bending cycles.

ince the advent of Bronze Age (c. 3600-1200 BCE), metal forming is carried out at temperatures close to melting where the yield stress of a metal is low and the metal becomes 'softer', thus amenable to shaping into desired forms. This principle, termed as 'hot-working' in metallurgy, is the backbone of all the modern day metal forming processes ${ }^{1}$ to produce not only day-to-day articles but also intricate metal structures involving hierarchical features with mixed length scales, albeit at macroscale. On the other hand, shaping of metals on a micrometer scale (and below) is conventionally carried out using lithographic techniques where a sacrificial polymeric resist pattern is made over which a metal film is deposited, and subsequently, the resist is removed by "lift-off". Unlike in the case of hot-working, producing hierarchical patterns on micron or sub-micron scale using simple lithography processes is not straightforward. Nonetheless, globally there is an intense research activity using lithography processes to produce ordered metal nanostructures which are of immense interest in plasmonics $^{2}$, magnetic ${ }^{3}$, catalytic ${ }^{4}$ and other applications. For producing metal surfaces with hierarchical patterning (MSHPs), direct laser writing ${ }^{5}$ and $\mathrm{x}$-ray lithography ${ }^{6}$ have been the methods of choice on thick resists which is then translated to form a MSHP ${ }^{7,8}$. For layer-by-layer patterning, optical and electron beam lithography techniques have been employed ${ }^{9}$. Undeniably, it is a huge challenge to produce MSHP using conventional lithography techniques.

Direct patterning of metals via solution processable precursor routes that are amenable to lithography is an attractive alternative to conventional lithography techniques as it reduces the number of process steps. Till date, such routes using metal-organics as lithography resists, have been employed for simple two-dimensional patterning of metals by direct write electron beam lithography ${ }^{10,11}$ and micromolding ${ }^{12,13}$. For large area patterning, nanoimprint lithography (NIL) is ideal ${ }^{14}$, although direct imprinting of metal patterns has not been attempted thus far; there are examples related to metal oxides using precursors such as metal methacrylates ${ }^{15}$ and alkoxides $^{16-18}$. These precursors require polymer additives to make them imprintable, and the latter once cross-linked, are difficult to get rid of unless heated to a higher temperature. Another way to pattern metals is to imprint nanoparticle inks directly and sinter the particles to obtain continuous structure ${ }^{19,20}$, though sintering usually leads to inferior properties of the imprinted features. The present work is an attempt to fix such problems associated with direct write recipes in the context of NIL, which has been performed taking palladium (Pd) as an example. Our process utilizes the principle of hot working to shape the metal organic ink close to its melting temperature whilst lithography is used to produce three-dimensional hierarchical patterns, layer-by-layer stacking of metal patterns and embedding Pd structures in a flexible substrate. The entire process was carried out well below the melting point of Pd and without the "lift-off" step. 


\section{Results}

The two important criteria that a metal-organic ink is required to qualify for direct NIL are the following: Firstly, it should have a welldefined transition temperature, $\mathrm{T}$ (analogous to $\mathrm{T}_{\mathrm{g}}$ in the case of polymers), above which it softens and flows smoothly into micro/ nano channels of the imprint mold (Figure 1), and secondly, it should undergo direct transformation to the desired nanomaterial under gentle thermal conditions. These aspects have been examined in respect of the chosen precursor Pd benzylthiolate prior to direct nanoimprinting. Thermogravimetric analysis (TGA) (Supplementary Figure S1) showed that the residue resulting from the loss reached a constant value of $43 \%$ beyond $250^{\circ} \mathrm{C}$, which, assuming that the residue corresponds to Pd metal, nearly matches with the expected weight loss (45\%). Indeed, x-ray diffraction (XRD) of a thermolysed film showed crystalline Pd peaks with the film being relatively smooth as shown in the SEM image (Supplementary Figure S1). Thus, direct precursor to metal conversion was realized. Differential scanning calorimetry (DSC) of the precursor showed a sharp endothermic peak at $\sim 120^{\circ} \mathrm{C}$ (Supplementary Figure S1), coinciding with melting of the Pd benzylthiolate (cycle 1). In repeated melt-cool cycles, the precursor showed DSC melting traces that are much shallower (Supplementary Figure S1). The irreversible nature of the melting transition in the present case plays an important role in preserving the integrity of the patterns during thermolysis. At the same time, second shallow (i.e., surface) melting can be employed in creating interesting and versatile hierarchical structures.
Direct two-dimensional nanoimprinting of Pd. Pd benzylthiolate dissolves well in many common organic solvents such as toluene, chloroform, acetone, etc., and forms smooth films when spin-coated (among others, chloroform solution gave the best films). The thickness of the film is highly controllable by varying the concentration of the precursor and the spin speed (Supplementary Table S1). The spin-coated Pd benzylthiolate film was imprinted by applying a pressure of $\sim 50$ bar close to its melting temperature $\left(\sim 120^{\circ} \mathrm{C}\right)$ using a Si mold with $250 \mathrm{~nm}$ grating structures. During imprinting, the molten precursor filled the channels in the Si mold. After the imprinting process, the setup was cooled down to room temperature for demolding. Post-demolding, Pd benzylthiolate patterns on the substrate were obtained. Henceforth, the process is called as melting-induced direct NIL (MD-NIL). The as-imprinted patterns were $\sim 210 \mathrm{~nm}$ wide (Figures $2 \mathrm{a}$ and $\mathrm{b}$ ) with a thickness of $\sim 185 \mathrm{~nm}$ (Figure $2 \mathrm{~b}$ and Supplementary Figure S2). After thermolysis at $250^{\circ} \mathrm{C}$ for $1 \mathrm{hr}$, the obtained Pd patterns shrunk to $\sim 100 \mathrm{~nm}$ (Figures $2 \mathrm{c}$ and $\mathrm{d}$ ), with a thickness of $\sim 70 \mathrm{~nm}$ (Figure $2 \mathrm{~d}$ and Supplementary Figure S2). In other words, the lateral (47\%) and vertical shrinkages (38\%) were due to the loss of the organic moieties. Thus, the distance between the features increased, while the pitch of the grating remained the same. An excellent imprinting yield of nearly 100\% was obtained and this can be clearly seen in the photograph of the imprinted features $\left(\sim 2 \mu \mathrm{m}\right.$ ) over an area of $2 \times 2 \mathrm{~cm}^{2}$ (Figure $2 \mathrm{l}$ and Supplementary Figure S3). The diffracted color of the ambient light shows large scale homogeneity across the patterned area. Various

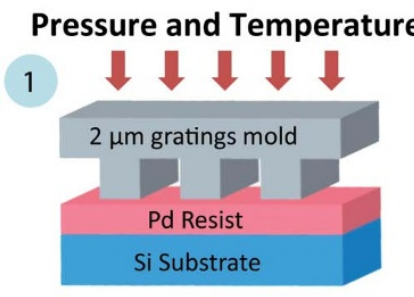

$\downarrow$

\section{Imprint on Molten Resist}

2

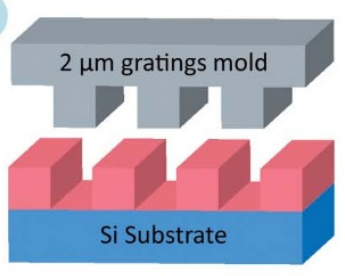

3

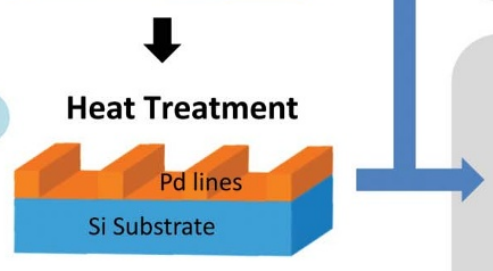

Hierarchical Patterning

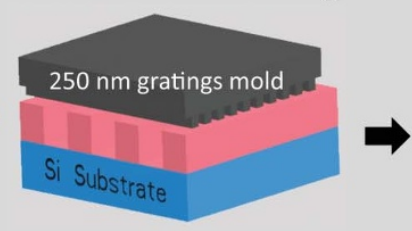

(a)
3D Hierarchical Structures

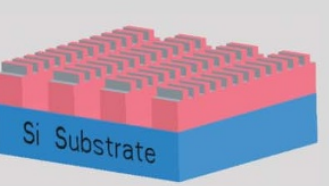

(b)

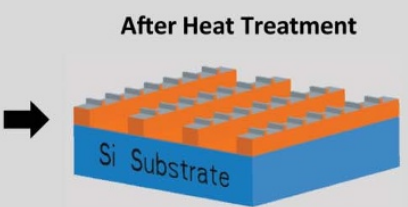

(c)

\section{Transfer Stacking}

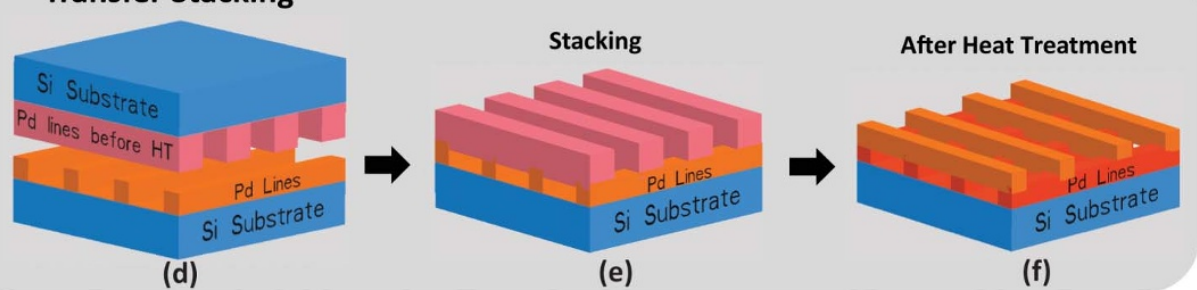

(d)

(e)

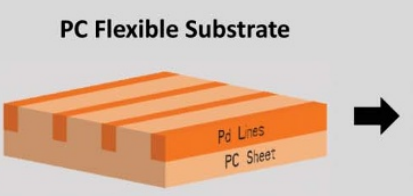

(h)

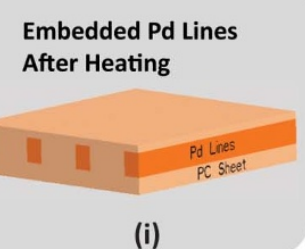

Embedded Pd Lines

PC Transfer

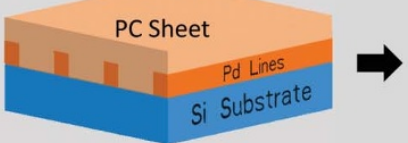

(g)

Figure 1 Schematic illustration of the MD-NIL. (1) The spin coated Pd benzylthiolate film was imprinted using a Si mold (possessing $\sim 2 \mu \mathrm{m}$ grating structures) close to its melting temperature $\left(\sim 120^{\circ} \mathrm{C}\right)$ by applying a pressure of $\sim 50$ bar. (2) During imprinting, the molten precursor flowed into and filled the channels in the Si mold, following which the setup was cooled down. (3) After demolding, Pd benzylthiolate patterns on the substrate were heat treated at $250^{\circ} \mathrm{C}$ for $1 \mathrm{hr}$ to obtain Pd patterns. At (2) stage, (a) hierarchical patterning can be done using a different Si mold with smaller feature sizes kept at right angles to the imprinted Pd benzylthiolate patterns. (b) Second MD-NIL leads to hierarchical Pd benzylthiolate patterns, which was heat treated (c). Transfer stacking - (d) using (2) as a mold and (3) as a substrate placed at right angles with respect to orientation of Pd lines, Pd benzylthiolate lines can be (e) transfer stacked by MD-NIL and (f) heat treated to get Pd crossbars. Polycarbonate (PC) transfer - (g) using (3) as mold and PC as substrate, imprinting by subjecting PC to glass transition $\left(\mathrm{T}_{\mathrm{g}}\right)$, (h) following cooling, demolding led to PC carrying Pd lines, (i) which was heated on a hot plate to embed the Pd lines underneath PC surface. 

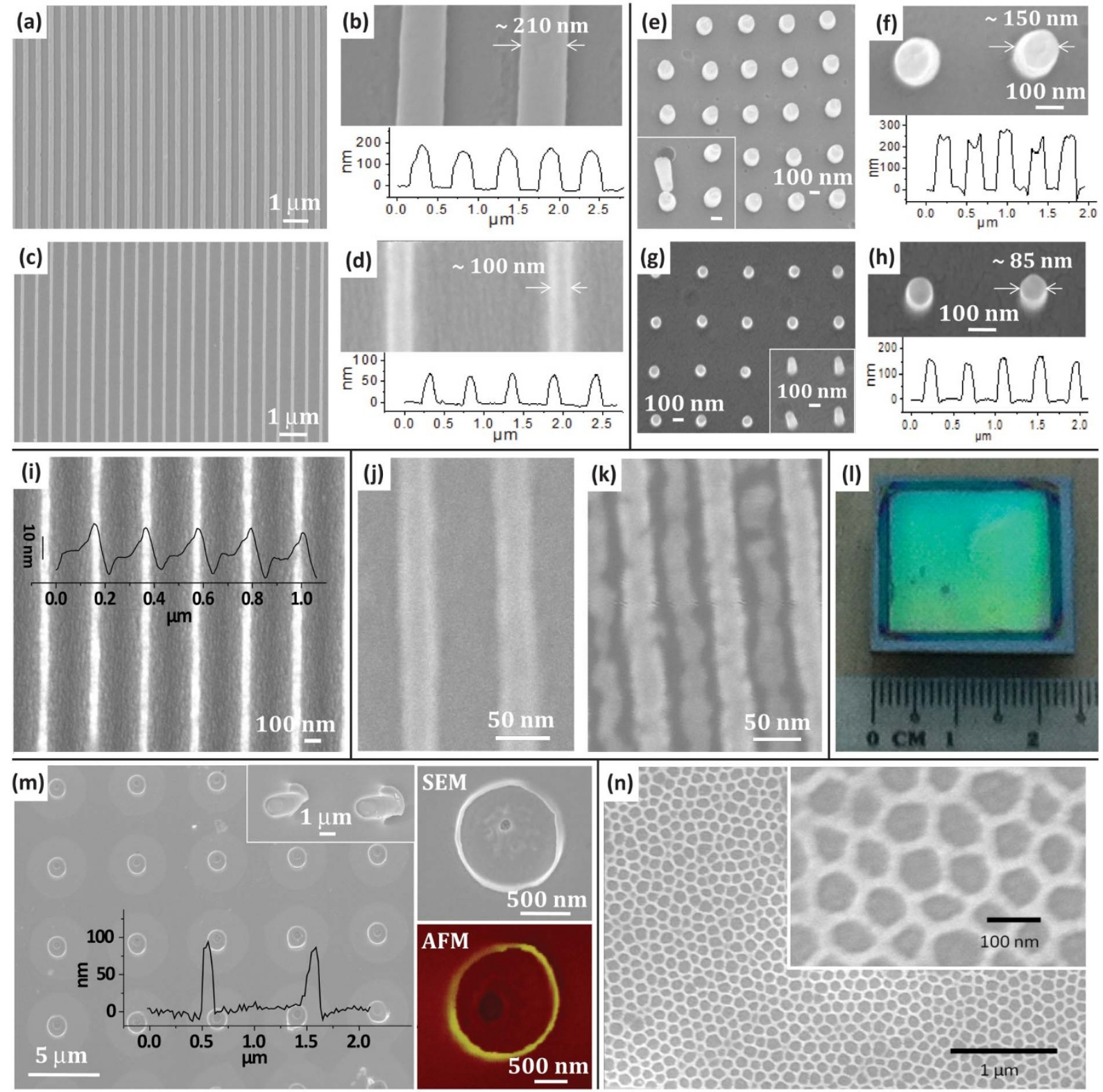

Figure 2 Direct nanoimprinting of Pd down to sub-50 $\mathbf{n m}$ resolution. (a,c) SEM images of the as-imprinted and heat treated lines of Pd benzylthiolate with (b,d) showing magnified views and the AFM $z$-profiles below respectively. SEM image of the Pd pillars of high aspect ratio (e) before and (g) after heat treatment with the magnified SEM images and $z$-profiles in (f) and (h) respectively. (i) SEM image of the imprinted Pd features using $100 \mathrm{~nm}$ mold after heat treatment. AFM $z$-profile is overlaid. SEM images of $35 \mathrm{~nm}$ line features (j) as-imprinted and (k) after heat treatment. (l) Digital photograph of uniformly imprinted pattern of $2 \mu \mathrm{m}$ Pd gratings over large area, i.e., $2 \times 2 \mathrm{~cm}^{2}$. SEM image of the $(\mathrm{m})$ Pd nanotubes after heat treatment with AFM $z$ profile overlaid; magnified SEM and AFM images shown alongside (Inset shows tilted SEM image of the nanotubes before heat treatment). (n) Hexagonal hole array of Pd after heat treatment. The minimum wall size between the holes after heat treatment is $\sim 23 \mathrm{~nm}$.

morphologies can be directly imprinted by suitably selecting the Si mold (Supplementary Figures S4 and S5). Circular tall pillars of aspect ratio close to 2 were patterned (Figures 2e and f) using similar imprinting procedure (Supplementary Table S1). The imprinted pillars $(\sim 260 \mathrm{~nm}$ tall, $\sim 150 \mathrm{~nm}$ wide) were intact and the aspect ratio is vividly seen in the case of a broken pillar shown in the inset (Figure 2e). After heat treatment, the pillar height decreased to $\sim 160 \mathrm{~nm}$ and the width became $\sim 85 \mathrm{~nm}$ (Figures $2 \mathrm{~g}$ and $\mathrm{h}$ ). The present method of direct imprinting does not take away high resolution achievable by NIL. Imprinted Pd lines of $\sim 40 \mathrm{~nm}$ width (after shrinkage) can be obtained routinely (Figure $2 \mathrm{i}$ and Supplementary Figure S6). Using a mold with feature size $\sim 35 \mathrm{~nm}, \mathrm{Pd}$ nanolines of width $\sim 30 \mathrm{~nm}$ were obtained (Figures $2 \mathrm{j}$, $\mathrm{k}$ and Supplementary Figure S7). The residual layer between the Pd features can be seen in the image (Figure $2 \mathrm{k}$ ) as it was not optimized to be devoid of residual layer at this point of time. It must be emphasized that the thickness of residual layer can be effectively controlled or eliminated completely by adjusting the concentration of the precursor solution. When the film thickness is more than the feature depth in Si mold, the residual or scum layer is always present. In order to produce isolated $\mathrm{Pd}$ features without the scum layer, the concentration of Pd benzylthiolate solution was varied so as to produce a thin film with thickness $(\sim 500 \mathrm{~nm})$ less than the height of the recessed features $(\sim 2 \mu \mathrm{m})$ of the Si mold (Supplementary Table S1). Upon imprinting, due to capillary action of the mold, the precursor Pd benzylthiolate filled the mold, while leaving the gaps between the recessed features. This phenomenon draws an analogy with capillary force lithography which employs similar 
concept for imprinting isolated polymer features ${ }^{21}$. An advantage of the present method is that there is no need of breakthrough etch, as is usually required in $\mathrm{NIL}^{22}$. Hollow $\mathrm{Pd}$ nanotubes can also be imprinted directly using an appropriate mold (optical image in Supplementary Figure S8). The SEM and AFM images in Figure $2 \mathrm{~m}$ show the wall width of the nanotube as $\sim 100 \mathrm{~nm}$ with a height of $\sim 90 \mathrm{~nm}$ (overlaid AFM profile in Figure $2 \mathrm{~m}$ ) after heat treatment. The aspect ratio and morphology of the as-imprinted nanotube can be seen clearly in a tilted view (inset in Figure $2 \mathrm{~m}$ ). Uniform filling of the molten Pd precursor is the key for the successful imprinting of various morphologies including nanotubes. Similarly, hexagonal nano-hole arrays of $\mathrm{Pd}$ can be uniformly imprinted over large areas (Figure $2 \mathrm{n}$ and Supplementary Figure S9). They possess an average wall width of $\sim 23 \mathrm{~nm}$ after the heat treatment.

Direct three-dimensional hierarchical nanoimprinting and transfer stacking of Pd nanopatterns. The direct MD-NIL method presented here can be employed for hierarchical patterning of $\mathrm{Pd}$ (see schematic in Figures 1a-c) by taking advantage of the hysteretic melting behavior of the Pd benzylthiolate precursor (Supplementary Figure S1). This is in contrast to hierarchical patterning of polymers by NIL, which was achieved by sequential imprinting steps ${ }^{23}$; the second imprinting is usually done below $\mathrm{T}_{\mathrm{g}}$. Hierarchical patterning with MD-NIL is illustrated in Figure 3, wherein a primary grating (line width $\sim 2 \mu \mathrm{m}$ ) was used as a primary mold to imprint Pd benzylthiolate line gratings, over which finer gratings $(\sim 160 \mathrm{~nm})$ were imprinted using a secondary mold (Figure 3a). The temperature, pressure and time of imprinting were optimized such that the primary structure did not collapse while imprinting the secondary structure on top of it (Supplementary Figure S10). Following heat treatment, the secondary lines were $\sim 50 \mathrm{~nm}$ wide and $\sim 25 \mathrm{~nm}$ tall over the primary line gratings of width $\sim 1.04 \mu \mathrm{m}$ (see Supplementary Figure S11 for large area SEMs). The shrinkage was not exactly the same as was observed for simple two-dimensional imprinting (Figure 2) as the imprinting conditions were slightly different.

Stacked patterns of the Pd gratings (Figures $3 \mathrm{~b}-\mathrm{g}$ ) can also be created by the MD-NIL method. Initially, Pd benzylthiolate was imprinted using a $\sim 2 \mu \mathrm{m}$ grating mold and heat treated to obtain Pd patterns. In the second imprinting step, instead of using a Si mold, another as-imprinted (without heat treatment) grating of Pd benzylthiolate $(2 \mu \mathrm{m})$ was used as a mold for imprinting (see schematic in Figures $1 \mathrm{~d}-\mathrm{f})$. Upon imprinting under the optimal applied pressure $\left(25\right.$ bar) and temperature $\left(130^{\circ} \mathrm{C}\right)$, the $\mathrm{Pd}$ benzylthiolate grating on the second mold melted and got transferred to the bottom substrate (Figure 3b; see Supplementary Figure S12 for large area optical images). The second stack of the Pd benzylthiolate does not
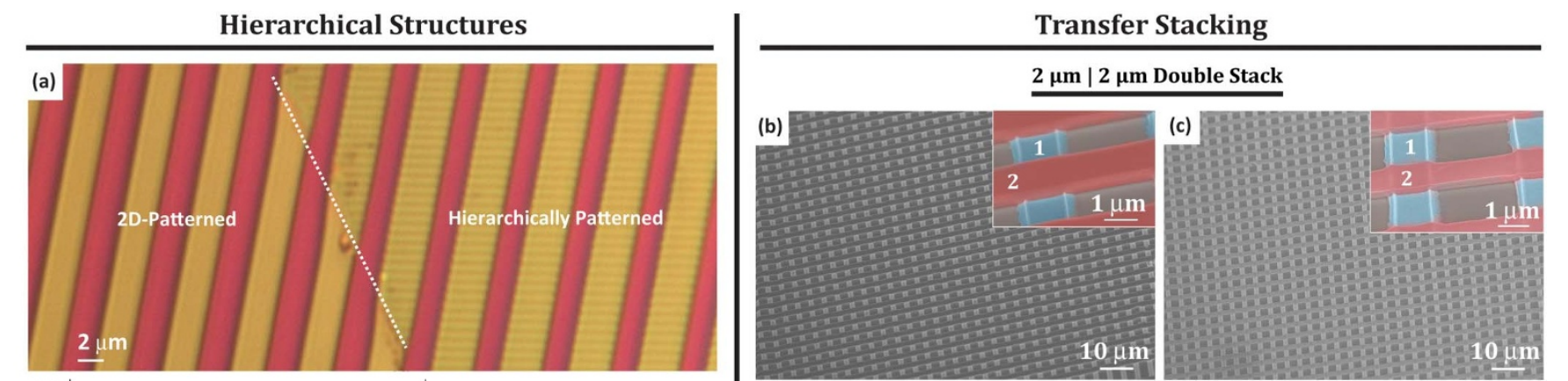
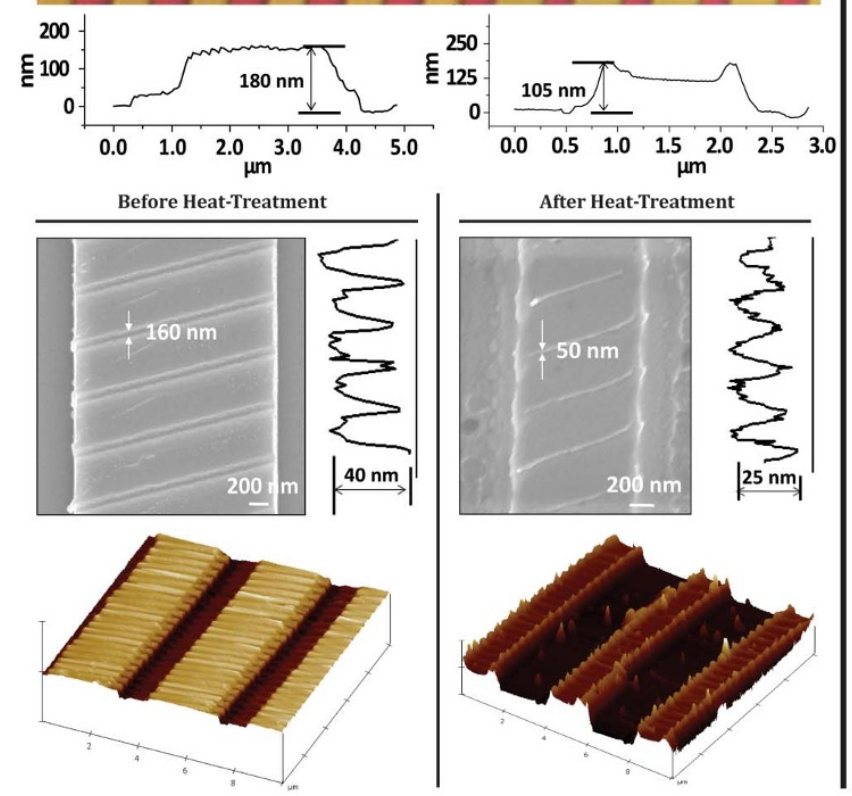
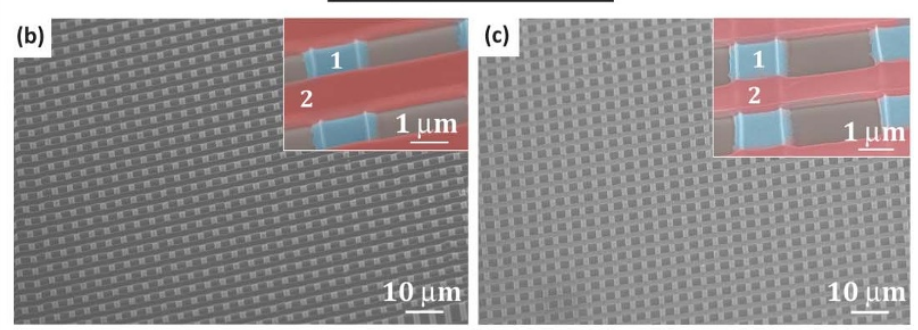

$2 \mu \mathrm{m}|2 \mu \mathrm{m}| 2 \mu \mathrm{m}$ Triple Stack
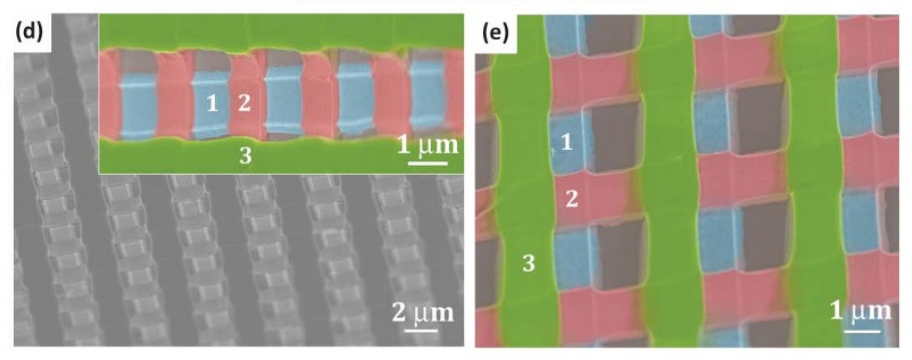

$250 \mathrm{~nm}$ | $250 \mathrm{~nm}$ Double Stack

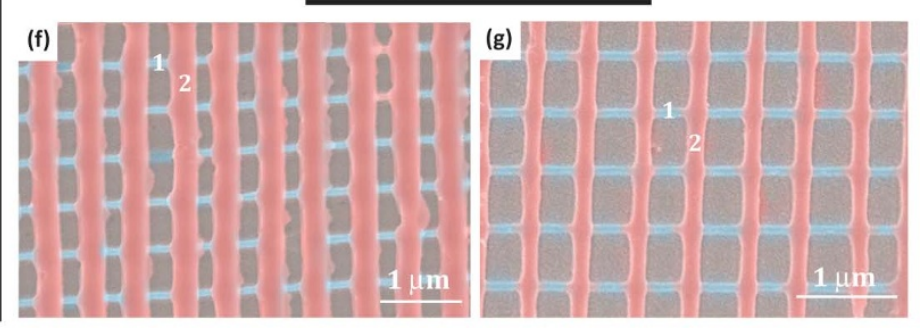

Figure 3 | (a) Optical image demonstrating large area Pd hierarchical structures with a primary mold of $2 \mu$ m gratings and secondary mold of $250 \mathrm{~nm}$ gratings. SEM and AFM images of Pd hierarchical structures - before heat treatment (to the left) widths of primary lines are $2 \mu \mathrm{m}$ and secondary lines are $160 \mathrm{~nm}$ with corresponding heights of $180 \mathrm{~nm}$ and $40 \mathrm{~nm}$, respectively. After heat treatment (to the right), the primary lines are $1.04 \mu \mathrm{m}$ wide with secondary lines of $50 \mathrm{~nm}$, and corresponding heights are $105 \mathrm{~nm}$ and $25 \mathrm{~nm}$, respectively. (b-g) Transfer stacking - (b) SEM image of the transfer stacked $2 \mu \mathrm{m}$ Pd lines and (c) after heat treatment. (d) Transfer stacked $3^{\text {rd }}$ stack in a tilted view; inset shows magnified image and (e) after heat treatment. A $250 \mathrm{~nm}$ grating stacked over another $250 \mathrm{~nm}$ grating (f) before and (g) after heat treatment. Images are false colored for illustration; blue color ( $1^{\text {st }}$, i.e., primary line grating), light red color $\left(2^{\text {nd }}\right.$ stack $)$ and green color $\left(3^{\text {rd }}\right.$ stack $)$. 
touch the substrate surface which is evident from the AFM profile (Supplementary Figure S13). Thus obtained patterns after transfer stacking can be subjected to heat treatment (Figure 3c). Another round of transfer stacking was performed using double stacked Pd lines as a substrate and freshly imprinted Pd thiolate grating as a mold (Figures $3 \mathrm{~d}$ and e). The transfer stacking is possible with smaller features as well. Figures $3 \mathrm{f}$ and g illustrate a $250 \mathrm{~nm}$ Pd grating stacked over a bottom $250 \mathrm{~nm}$ Pd grating, as-imprinted and after the heat treatment, respectively. Here, an analogy can be drawn with the adhesion driven transfer printing ${ }^{24}$ where the adhesion of the transferred nanostructures can be kinetically switched between the host and the substrate. In the present case, the transfer stacking is mediated due to the melting of Pd benzylthiolate which facilitates the transfer to the bottom Pd lines. Importantly, the procedure is reproducible and can be extended to even finer features. It may be noted that the imprinting pressure chosen for transfer stacking is an important parameter as excess pressure can cause the top lines to sag towards the substrate leading to their cracking (Supplementary Figure S14).

A preliminary measurement of reflectance of the Pd double stack $(250 \mathrm{~nm} \times 250 \mathrm{~nm})$ in comparison to controls i.e., Pd film and gratings, has shown that double stack possesses increased reflectance (Figure S15). For Pd double stack, the reflectance reached $\sim 100 \%$ at about $\sim 2 \mu \mathrm{m}$, whereas for Pd film and gratings, the reflectance values were far below. First of all, it is not surprising to see the stop band with 2-layers, as the band gap shows up even for a double grating in metallic photonic crystals ${ }^{25}$. Although complete band gap was not observed, band edge (where the reflectance reaches 50\%) was noted to be $0.65 \mu \mathrm{m}$. Also, a small dip was observed in lower wavelength regime of Pd double stack structure (Figure S16). In the literature, periodic grating structures (double or single layer) are known to excite the surface plasmon polaritons (SPPs) ${ }^{25,26}$. Although Pd is a weak plasmonic metal, the effect of SPPs is clearly observable ${ }^{27}$, more so in angle-resolved spectra ${ }^{28}$. In the visible wavelength regime $(<2$ $\mu \mathrm{m})$, by varying the angle, it was observed that the dip does change the wavelength position but not the peak intensity or amplitude, which further reaffirms the excitation of $\operatorname{SPPs}^{28}$.

Anisotropic wetting - hierarchically patterned hydrophobic metal surfaces. An interesting possibility with a MSHP is its tunable surface wettability. A superhydrophobic metal surface, for instance, finds crucial applications in heavy machinery and automotive industries ${ }^{29}$. However, realizing a superhydrophobic metal surface is rather difficult given that the metal surfaces are generally hydrophilic ${ }^{30}$ due to the polar nature of the thin oxide overlayers, which get formed in the ambient ${ }^{31}$. The hydrophobic nature is imparted to metal surfaces routinely by replacing the oxide overlayers with self-assembling molecular monolayers ${ }^{32}$ or by coating with polymer layers ${ }^{33}$, made of saturated, non-polar groups. These coatings may wear off due to their limited life-time, however while present, they prohibit direct access to the metal surface or cause significant chemical modification. Physical methods such as plasma etching ${ }^{34}$, laser ablation ${ }^{35,36}$ and growing metal nanostructures ${ }^{37}$ have also been employed, which in general, produce disordered structures with some increase in hydrophobicity. MD-NIL can be a versatile method for producing hydrophobic metal surfaces without compromising the chemical purity, stability and edge roughness of the patterns. Going beyond the usual hydrophobic surface, MSHPs with typical feature length scales below $\sim 1 \mu \mathrm{m}^{38}$ may be explored for anisotropic wetting ${ }^{39}$, wherein the contact angles along different directions on the surface may differ significantly ${ }^{40}$. The anisotropic wettability is generally qualified by the difference in static contact angles of water droplets measured in two directions, parallel and perpendicular to the stripes. Butterfly wing ${ }^{41}$ and rice leaf ${ }^{42}$ are examples from nature exhibiting such a property. Natural rice leaf possesses papillae of about $5-8 \mu \mathrm{m}$ arranged in parallel with innumerable pins of nanometer scale, which bring in the anisotropic wetting property ${ }^{43}$. Using MD-NIL, a MSHP mimicking rice leaf structure, i.e., metallic rice leaf, was obtained by first imprinting a primary grating with a $2 \mu \mathrm{m}$ mold, over which fine dimples $(\sim 200 \mathrm{~nm})$ were patterned (Figure $4 \mathrm{a}$ and see Supplementary Figure S17 for SEM and AFM images). The water contact angle of the as-imprinted rice leaf structure was found to be $137^{\circ}$, which is comparable to that of a natural rice leaf ${ }^{44}$. After thermolysis, a Pd MSHP resulted and the contact angle in this case was surprisingly, only slightly lower $\left(\sim 130^{\circ}\right)$, although the underlying surface had turned metallic and hydrophilic! More importantly, the hierarchical patterns are well-ordered and smooth, unlike the ones produced by laser flashing ${ }^{29}$. In clear contrast to the behavior of the hierarchical pattern, the line patterns alone (without the second imprint) exhibited a much lower contact angle of $90.1^{\circ}$, and similar was the situation with only the dimple patterns $\left(88^{\circ}\right)$ (Supplementary Figure S18). For natural rice leaf, the values are $138.5^{\circ}$ and $131.7^{\circ}$ respectively $^{44}$. For the metallic rice leaf fabricated here, the contact angles were $130^{\circ}$ and $92^{\circ}$, respectively, in parallel and perpendicular directions, thus clearly depicting the anisotropic wetting. It is clear that the hierarchical structuring not only increases overall hydrophobicity but also gives rise to anisotropic wetting. In order to further increase the hydrophobicity, a monolayer of hexadecanethiol molecule was adsorbed onto the metallic rice leaf by immersion in $1 \mathrm{mM}$ of the thiol solution in ethanol. After $3 \mathrm{hrs}$ of immersion, the substrate was taken out, rinsed and then the contact angles were measured. As can be seen from Figure $4 \mathrm{~b}$, the contact angles increased in both parallel and perpendicular directions to $152^{\circ}$ and $125^{\circ}$ respectively. Another important characteristic of hierarchical structures is that they can enhance the Raman signal of molecules adsorbed ${ }^{45,46}$. Palladium hierarchical structure is also found to enhance the Raman signal of the adsorbed thiol molecules (Figure 4c). The enhancement factor calculated taking into account the $v(\mathrm{C}-\mathrm{S})_{\text {trans }}$ stretch, following the procedure suggested by $\mathrm{Yu}$ et $\mathrm{al}^{47,48}$, is 0.36 $\times 10^{5}$ (see Supplementary S19 for calculation).

Transfer imprinting to flexible substrates. While flat inflexible substrates are relatively straightforward to use as substrates in direct nanoimprinting, flexible substrates carrying such metal patterns, although difficult to process, may find many interesting applications. To name a few, macroelectronics opens up new avenues while designing circuits for electronic textiles ${ }^{49}$, sensory skins $^{50,51}$, liquid crystal displays or $\mathrm{x}$-ray imaging devices ${ }^{52,53}$. However, when flexible substrates are subjected to high temperature heat treatment (during thermolysis of the precursor), they undergo degradation as they cannot withstand the temperature. In fact, this factor limits the imprintability of various direct write precursors onto flexible substrates owing to high processing temperatures. In order to circumvent this problem, we developed the transfer imprinting method. This method involves transferring the fabricated Pd patterns after processing from a rigid substrate to a flexible substrate. Here the flexible substrate chosen was polycarbonate (PC) which possesses a glass transition temperature, $\mathrm{T}_{\mathrm{g}} \sim 150^{\circ} \mathrm{C}$. NIL was performed by using the thermolysed $\mathrm{Pd}$ patterns on $\mathrm{Si}$ (Figure $5 \mathrm{a}$ and $\mathrm{b}$ ) as a mold (see schematic $3 \mathrm{~g}-\mathrm{i}$ in Figure 1 ) and $\mathrm{PC}$ as substrate. Residual layer free Pd patterns were chosen for this purpose. While imprinting, the capillary action is more prominent along the walls of the recessed features of Si mold, which leads to raised sides of the $\mathrm{Pd}$ features as discernible from the cross section SEM (Figure 5a). When the PC substrate was taken through $\mathrm{T}_{\mathrm{g}}$, softened PC flowed into the channels thus contacting the Pd lines all over. After cooling down of the setup, the PC substrate was demolded carrying the transferred $\mathrm{Pd}$ lines on its surface (Figure 5c). The Pd lines shown in cross-section in Figure 5d clearly illustrate that the PC film is in intimate contact with Pd on 

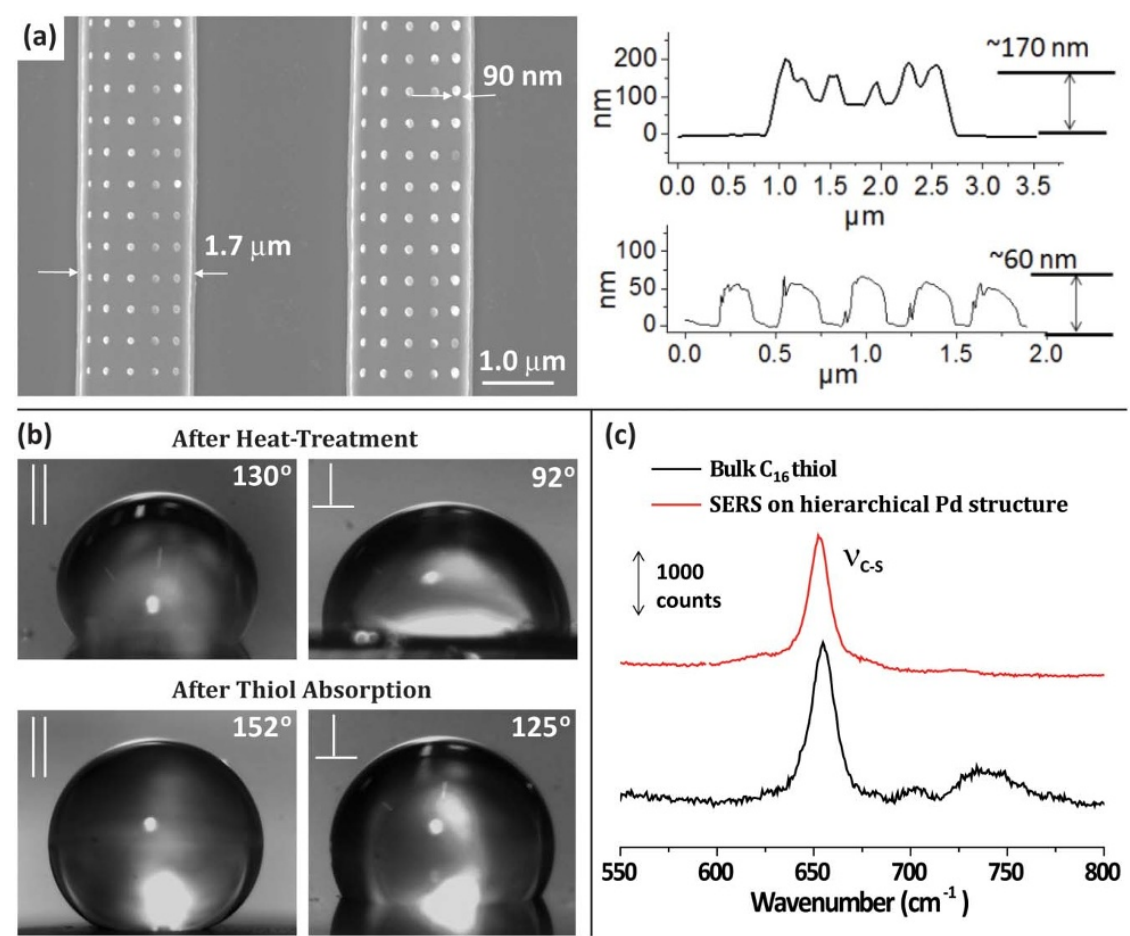

Figure $4 \mid$ Biomimicking. (a) SEM images of metallic rice leaf pattern (after heat treatment) with primary lines of $1.7 \mu \mathrm{m}$ and secondary pillars of $90 \mathrm{~nm}$, and corresponding height of $170 \mathrm{~nm}$ and $60 \mathrm{~nm}$, respectively, marked in the AFM profiles. (b) The contact angles in parallel and perpendicular direction of the metallic rice leaf lines differ due to anisotropic wetting. After hexadecanethiol absorption, the contact angle is increased in both directions.

(c) Surface enhanced Raman spectrum of a monolayer of the adsorbed thiol molecules on Pd rice leaf compared with that from a drop of neat thiol. The peak observed can be assigned to $v(\mathrm{C}-\mathrm{S})_{\text {trans }}$ stretch. The signal from the monolayer is of similar intensity as the bulk, due to SERS activity of the metallic rice leaf structure.
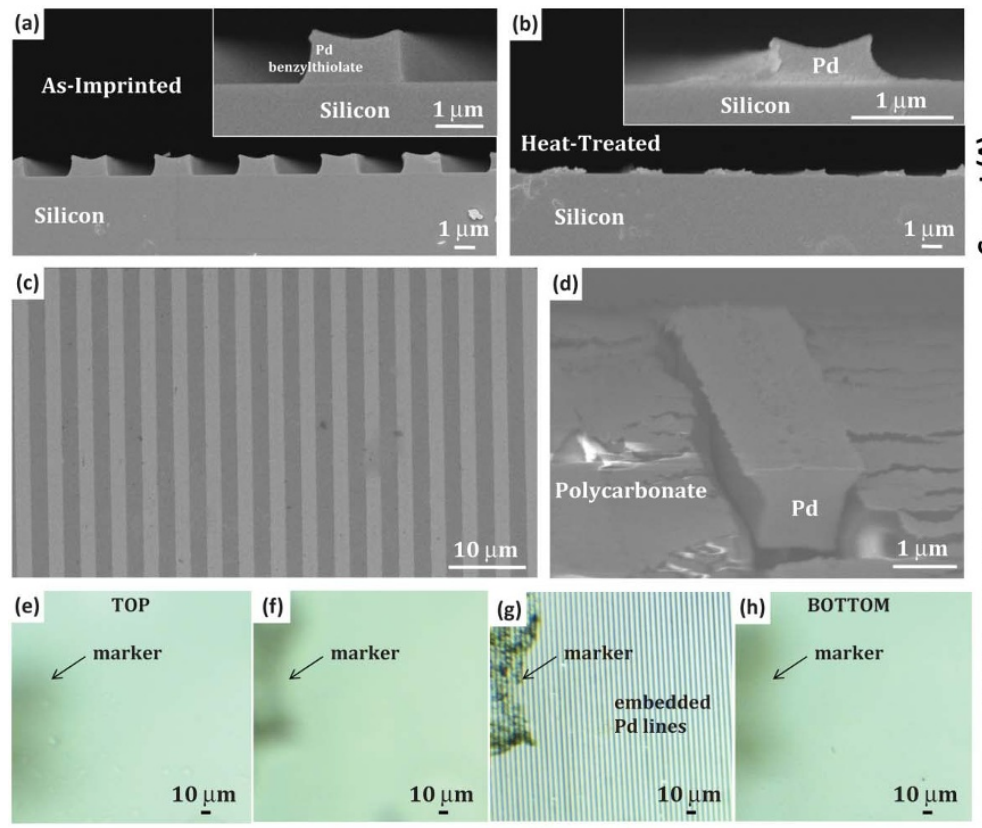
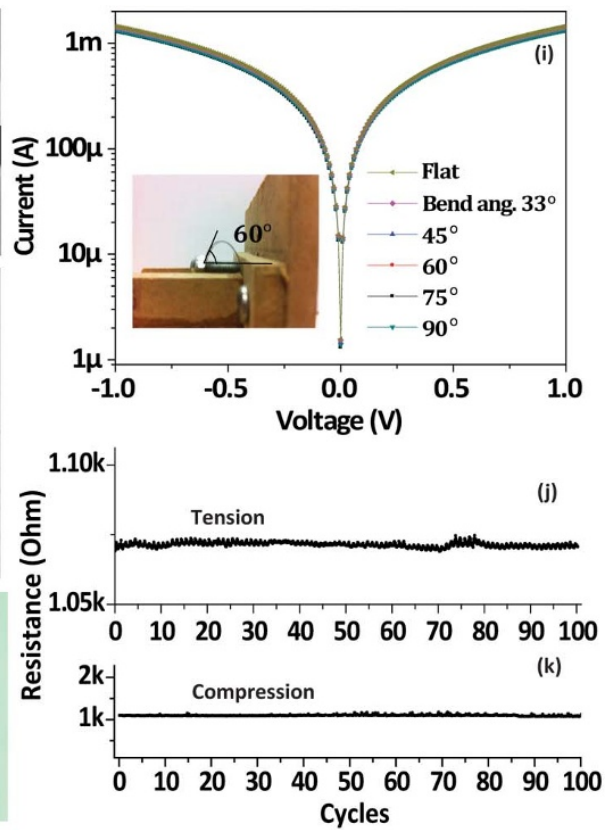

Figure 5 Transfer imprinting of Pd lines to flexible polycarbonate (PC) substrate. Cross-section SEM images of the $2 \mu \mathrm{m}$ Pd gratings (a) as-imprinted and (b) after heat treatment, with magnified views in the insets. (c) Pd lines transferred to PC substrate and (d) cross-sectional view showing a partially embedded Pd line into PC substrate (at a SEM tilt angle of $60^{\circ}$ ). The sample was coated with Au to avoid surface charging while imaging in SEM.

(e-h) Optical micrographs of the Pd lines embedded inside PC substrate. Images taken while different planes of the substrate were in optical focus, from top surface to the bottom surface of the PC. Arrow marks indicate the dust particle as an identification marker. (i) I-V characteristics of Pd lines in PC while bending to various angles, with the inset showing a digital photograph of the PC substrate with Pd lines at a bend angle of $60^{\circ}$. The resistance of Pd lines at a bend angle of $60^{\circ}$ for various cycles of (j) tensile and (k) compressive strain. 
all the three sides. Importantly, the transfer imprinting method differs from the widely employed transfer technique to PDMS substrate (which is rubbery and permeable to gases ${ }^{54}$ ) by thermal curing $^{55}$. Embedding the Pd lines under the PC surface was achieved by heating the PC substrate carrying the Pd lines above $\mathrm{T}_{\mathrm{g}}$ again, during which the PC flowed and covered the Pd lines all around thus embedding them. Due to charging effects from the insulating PC substrate, cross section SEM was not possible. However, optical images (Figures $5 \mathrm{e}-\mathrm{h}$ ) taken from the top of the PC surface did not show any Pd lines. While focusing beneath the surface of PC, gratings became visible, revealing the embedded Pd lines (Figure 5g). This transfer imprinting method is general and can be extended to transfer of any patterned materials to flexible substrates. The electrical characteristics of PC substrate carrying the transferred Pd lines on surface were tested by subjecting it to bending. The I-V characteristics of the Pd lines did not change while bending the substrate even to an extreme bend angle of $90^{\circ}$ (Figure 5i). To further check the stability of the Pd lines while bending, the device was subjected to bending cycles (at $60^{\circ}$ ) in tension and compression geometry (Figures $5 \mathrm{j}$ and $\mathrm{k}$ ). The resistance remained constant for over 100 cycles.

\section{Discussion}

We have employed MD-NIL as a technique to directly imprint sub$50 \mathrm{~nm}$ metal hierarchical nanostructures. This method employs melting of the precursor as the key to fill the capillary channels of the mold during imprinting. In turn, the hysteretic melting of the precursor, enables fabrication of MSHP, which exhibit anisotropic hydrophobic wetting. The contact angles were significantly increased due to the hierarchical structuring and they differed in parallel and perpendicular directions of the water droplet. This anisotropy was further increased by chemical modification of the surface, i.e., by adsorbing a monolayer of thiol. The hierarchical pattern also acts as a Raman signal enhancer due to the surface structuring. By tuning the imprinting parameters, our method has been shown to potentially create metal patterns resembling woodpile structures at the nanoscale, which are of much demand as photonic crystals ${ }^{56}$. The present method provides a unique capability to pattern large areas with nanodomains with almost $100 \%$ yield. This may find wide applications in photonics, owing to the optical beam sizes ranging few microns in general. Further, the metal patterns were transferred to a flexible PC substrate which sustained without degradation to repeated bend cycles.

\section{Methods}

Pd benzylthiolate preparation. Benzyl mercaptan, palladium (II) acetate and toluene were purchased from Fluka, Alfa Aesar and Sigma Aldrich, respectively. Equimolar amounts of benzyl mercaptan was mixed with a solution of Pd acetate in toluene and stirred for $15 \mathrm{hrs}$. The unreacted Pd acetate residue was settled using centrifugation. The supernatant was separated and subjected to low pressure distillation in a rotary evaporator to remove the solvent. For further purification, the product was washed with acetonitrile to remove unreacted mercaptan and left to dry in a vacuum oven at room temperature. The product, Pd benzylthiolate, was a brownish-red colored powder. It was dissolved in chloroform to produce desired concentration. TGA (TA Instruments Q500) was used to determine the decomposition temperature of Pd benzylthiolate, while DSC (TA Instruments Q100) for melting point and in turn the imprinting conditions.

MD-NIL. Si(100) substrates and molds were cleaned with piranha solution at $150^{\circ} \mathrm{C}$ for $2 \mathrm{hrs}$, followed by rinsing with deionized water and blow drying using a nitrogen gun. The substrates were kept in an oven at $100^{\circ} \mathrm{C}$ for drying purpose, while the $\mathrm{Si}$ molds were treated with perfluorodecyl-trichlorosilane for 5 hrs to reduce their surface energy in order to facilitate a clean demolding after imprinting. Various types of molds were used for imprinting, namely, $2 \mu \mathrm{m}, 250 \mathrm{~nm}, 100 \mathrm{~nm}$ and $30 \mathrm{~nm}$ gratings with equal line width and spacing, a dimple mold with $200 \mathrm{~nm}$ holes which were $400 \mathrm{~nm}$ deep and 20-50 nm self-assembled pillars. Imprint lithography was carried out in an Obducat imprinter (Obducat, Sweden). The Pd benzylthiolate solution (filtered using a $0.2 \mu \mathrm{m}$ pore size filter) was spin coated at a spin speed of 400-6000 rpm. During MD-NIL, Si mold was placed on top of the film and imprinted at elevated pressure (30-50 bar) and temperature $\left(120^{\circ} \mathrm{C}\right)$ for $\sim 30 \mathrm{~min}$ using the Obducat nanoimprinter (Obducat, Sweden). After imprinting, demolding was done carefully leaving behind the structures on the substrate. The structured films on substrates were subsequently transferred to a Carbolite chamber furnace and heat treated at $250^{\circ} \mathrm{C}$ (at a rate of $10^{\circ} \mathrm{C} / \mathrm{min}$ ) for $60 \mathrm{~min}$ to remove the organics leaving behind Pd with negligible amount of carbon. For transferring Pd nanostructures onto flexible polycarbonate (PC) substrate, NIL was performed with PC as substrate and nanostructured $\mathrm{Pd}$ on $\mathrm{Si}$ as mold at $150^{\circ} \mathrm{C}$ and 50 bars.

Characterization. The imprints were imaged by an optical microscope (Carl Zeiss), field-emission scanning electron microscope (JEOL JSM-6700F, FE-SEM) and an atomic force microscope (Nanoscope ${ }^{\circledR}$ IV multimode AFM). X-ray diffraction diffractometer (Bruker D8 General Area Detector Diffraction System GADDS, XRD) was utilized for studying the crystallinity of the Pd thin film. A Ramé-Hart digital contact angle (CA) goniometer was used to measure the surface wetting properties of hierarchical Pd films at room temperature. A deionized (DI) water droplet $(\sim 3 \mu \mathrm{L})$ was deposited gently on the sample surface using an automatic pipette, and a photograph of the water droplet was taken immediately with the goniometer camera. $\mathrm{CA}$ values were given by the DROPimage advanced software measurement. The CA values obtained from the software were cross-checked with the CA values measured manually using the printed photograph of the water droplet. An average was taken after a few point measurements. For each point, a few images were recorded and measured. For performing I-V measurements, a Keithley semiconductor characterization system model 4200-SCS was used. Metal contacts of Au were deposited by placing mask over the Pd lines on PC substrate and final contact to pads was made by means of silver paint. Raman spectra were recorded in the backscattering geometry using a $532 \mathrm{~nm}$ excitation from a diode pumped frequency doubled Nd:YAG solid state laser (model GDLM-5015L, Photop Swutech, China) and a custom built Raman spectrometer equipped with a SPEX TRIAX 550 monochromator and a liquid nitrogen cooled CCD detector (Spectrum One with CCD3000 controller, ISA Jobin Yvon).

1. Dieter, G. E. Mechanical Metallurgy. Ch. 15 McGraw-Hill Book Company, New York, 1988.

2. Tan, Y. et al. Versatile fabrication of intact three-dimensional metallic butterfly wing scales with hierarchical sub-micrometer structures. Angew. Chem. Int. Ed. 50, 8307-8311 (2011).

3. Ctistis, G. et al. Optical and magnetic properties of hexagonal arrays of subwavelength holes in optically thin cobalt films. Nano Lett. 9, 1-6 (2008).

4. Wang, R., Wang, C., Cai, W.-B. \& Ding, Y. Ultralow platinum loading high performance nanoporous electrocatalysts with nanoengineered surface structures. Adv. Mater. 22, 1845-1848 (2010).

5. Cheng, Y., Huang, T. \& Chieng, C. C. Thick-film lithography using laser write. Microsyst. Technol. 9, 17-22 (2002).

6. Innocenzi, P., Malfatti, L. \& Falcaro, P. Hard X-rays meet soft matter: When bottom-up and top-down get along well. Soft Matter 8, 3722-3729 (2012).

7. Rill, M. S. et al. Photonic metamaterials by direct laser writing and silver chemical vapour deposition. Nat. Mater. 7, 543-546 (2008).

8. Cao, Y.-Y., Takeyasu, N., Tanaka, T., Duan, X.-M. \& Kawata, S. 3D Metallic nanostructure fabrication by surfactant-assisted multiphoton-induced reduction. Small 5, 1144-1148 (2009).

9. Levinson, H. J. et al. SPIE Handbook of Microlithography, Micromachining and Microfabrication. The Society for Photo-optical Instrumentation Engineers, United States of America, 1997.

10. Bhuvana, T. \& Kulkarni, G. U. Highly conducting patterned Pd nanowires by direct-write electron beam lithography. ACS Nano 2, 457-462 (2008).

11. Nedelcu, M. et al. Fabrication of sub-10 nm metallic lines of low line-width roughness by hydrogen reduction of patterned metal-organic materials. Adv. Funct. Mater. 20, 2317-2323 (2010).

12. Radha, B. \& Kulkarni, G. U. A modified micromolding method for sub-100-nm direct patterning of Pd nanowires. Small 5, 2271-2275 (2009).

13. Radha, B., Kiruthika, S. \& Kulkarni, G. U. Metal anion-alkyl ammonium complexes as direct write precursors to produce nanopatterns of metals, nitrides, oxides, sulfides, and alloys. J. Am. Chem. Soc. 133, 12706-12713 (2011).

14. Schift, H. Nanoimprint lithography: An old story in modern times : A review. J. Vac. Sci. Technol., B 26, 458-480 (2008).

15. Lim, S. H., Saifullah, M. S. M., Hussain, H., Loh, W. W. \& Low, H. Y. Direct imprinting of high resolution $\mathrm{TiO}_{2}$ nanostructures. Nanotechnol. 21, 285303 (2010).

16. Kunitake, T. \& Lee, S.-W. Molecular imprinting in ultrathin titania gel films via surface sol-gel process. Anal. Chim. Acta 504, 1-6 (2004).

17. Park, H.-H. et al. Photo-induced hybrid nanopatterning of titanium dioxide via direct imprint lithography. J. Mater. Chem. 20, 1921-1926 (2010).

18. Ganesan, R. et al. Direct patterning of $\mathrm{TiO}_{2}$ using step-and-flash imprint lithography. ACS Nano 6, 1494-1502 (2012).

19. Liang, C.-C. et al. Plasmonic metallic nanostructures by direct nanoimprinting of gold nanoparticles. Opt. Express 19, 4768-4776 (2011).

20. Ko, S. H. et al. Direct nanoimprinting of metal nanoparticles for nanoscale electronics fabrication. Nano Lett. 7, 1869-1877 (2007).

21. Suh, K. Y. \& Lee, H. H. Capillary force lithography: large-area patterning, selforganization, and anisotropic dewetting. Adv. Func. Mater. 12, 405-413 (2002).

22. Chou, S. Y., Krauss, P. R. \& Renstrom, P. J. Imprint lithography with 25 nanometer resolution. Science 272, 85-87 (1996). 
23. Fengxiang, Z. \& Low, H. Y. Ordered three-dimensional hierarchical nanostructures by nanoimprint lithography. Nanotechnol. 17, 1884-1890 (2006).

24. Meitl, M. A. et al. Transfer printing by kinetic control of adhesion to an elastomeric stamp. Nat. Mater. 5, 33-38 (2006).

25. Yang, Y. L. et al. Fabrication and characterization of three-dimensional all metallic photonic crystals for near infrared applications. App. Phys. Lett. 94, 041122 (2009).

26. Li, Z. Y., El-Kady, I., Ho, K. M., Lin, S. Y. \& Fleming, J. G. Photonic band gap effect in layer-by-layer metallic photonic crystals. J. App. Phys. 93, 38-42 (2003).

27. Anttu, N., Guan, Z. Q., Hakanson, U., Xu, H. X. \& Xu, H. Q. Excitations of surface plasmon polaritons in double layer metal grating structures. App. Phys. Lett. 100, 091111 (2012).

28. Gao, H. et al. Rayleigh anomaly-surface plasmon polariton resonances in palladium and gold subwavelength hole arrays. Opt. Express 17, 2334-2340 (2009).

29. Water-Repelling Metals, http://www.technologyreview.com/energy/21530/ page1/, Date accessed: May 5, 2012.

30. Valette, G. Hydrophilicity of metal surfaces: Silver, gold and copper electrodes. J. Electroanal. Chem. Interfacial Electrochem. 139, 285-301 (1982).

31. Ojifinni, R. A. The Surface Chemistry of Atomic Oxygen Pre-covered Gold. The University of Texas at Austin, Austin, 2008.

32. Love, J. C., Estroff, L. A., Kriebel, J. K., Nuzzo, R. G. \& Whitesides, G. M. Selfassembled monolayers of thiolates on metals as a form of nanotechnology. Chem. Rev. 105, 1103-1170 (2005).

33. Levkin, P. A., Svec, F. \& Fréchet, J. M. J. Porous polymer coatings: A versatile approach to superhydrophobic surfaces. Adv. Funct. Mater. 19, 1993-1998 (2009).

34. Chao-Hua, X., Shun-Tian, J., Jing, Z. \& Jian-Zhong, M. Large-area fabrication of superhydrophobic surfaces for practical applications: An overview. Sci. Technol. Adv. Mater. 11, 033002 (2010).

35. Jagdheesh, R., Pathiraj, B., Karatay, E., Römer, G. R. B. E. \& Huisint Veld, A. J. Laser-induced nanoscale superhydrophobic structures on metal surfaces. Langmuir 27, 8464-8469 (2011).

36. Bieda, M., Beyer, E. \& Lasagni, A. F. Direct fabrication of hierarchical microstructures on metals by means of direct laser interference patterning. J. Eng. Mater. Technol. 132, 031015-031016 (2010).

37. Han, J. T. et al. Fabrication of a bionic superhydrophobic metal surface by sulfurinduced morphological development. J. Mater. Chem. 15, 3089-3092 (2005).

38. Liu, K. \& Jiang, L. Bio-inspired design of multiscale structures for function integration. Nano Today 6, 155-175 (2011).

39. Xia, D., Johnson, L. M. \& López, G. P. Anisotropic wetting surfaces with onedimesional and directional structures: Fabrication approaches, wetting properties and potential applications. Adv. Mater. 24, 1287-1302 (2012).

40. Xia, D., He, X., Jiang, Y.-B., Lopez, G. P. \& Brueck, S. R. J. Tailoring anisotropic wetting properties on submicrometer-scale periodic grooved surfaces. Langmuir 26, 2700-2706 (2010).

41. Zheng, Y., Gao, X. \& Jiang, L. Directional adhesion of superhydrophobic butterfly wings. Soft Matter 3, 178-182 (2007).

42. Feng, L. et al. Super-hydrophobic surfaces: From natural to artificial. Adv. Mater. 14, 1857-1860 (2002).

43. Wu, D. et al. Three-level biomimetic rice-leaf surfaces with controllable anisotropic sliding. Adv. Funct. Mater. 21, 2927-2932 (2011).

44. Zhu, D. et al. Mimicking the rice leaf-from ordered binary structures to anisotropic wettability. Langmuir 26, 14276-14283 (2010).

45. Goldberg-Oppenheimer, P., Mahajan, S. \& Steiner, U. Hierarchical electrohydrodynamic structures for surface-enhanced Raman scattering. $A d v$. Mater. 24, OP175-OP180 (2012).
46. Bhuvana, T. \& Kulkarni, G. U. A SERS-active nanocrystalline Pd substrate and its nanopatterning leading to biochip fabrication. Small 4, 670-676 (2008).

47. Yu, H.-Z., Zhang, J., Zhang, H.-L. \& Liu, Z.-F. Surface-enhanced Raman scattering (SERS) from azobenzene self-assembled "sandwiches". Langmuir 15, 16-19 (1998).

48. Bhuvana, T., Kumar, G. V. P., Kulkarni, G. U. \& Narayana, C. Carbon assisted electroless gold for surface enhanced Raman scattering studies. J. Phys. Chem. C 111, 6700-6705 (2007).

49. Sun, Y. \& Rogers, J. A. Inorganic semiconductors for flexible electronics. Adv. Mater. 19, 1897-1916 (2007).

50. Nathan, A. \& Chalamala, B. R. Special issue on flexible electronics technology, Part II: Materials and devices. Proc. IEEE 93, 1391-1393 (2005).

51. Nathan, A. \& Chalamala, B. R. Special issue on flexible electronics technology, Part I: Systems and applications. Proc. IEEE 93, 1235-1238 (2005).

52. Baca, A. J. et al. Semiconductor wires and ribbons for high- performance flexible electronics. Angew. Chem. Int. Ed. 47, 5524-5542 (2008).

53. Reuss, R. H. et al. Macroelectronics: Perspectives on technology and applications. Proc. IEEE 93, 1239-1256 (2005).

54. Sadrzadeh, M., Shahidi, K. \& Mohammadi, T. Synthesis and gas permeation properties of a single layer PDMS membrane. J. Appl. Polym. Sci. 117, 33-48 (2010).

55. Lee, C. H., Kim, D. R. \& Zheng, X. Fabricating nanowire devices on diverse substrates by simple transfer-printing methods. Proc. Natl. Acad. Sci. 107, 9950-9955 (2010).

56. Hillebrand, R. \& Gösele, U. Woodpiles for photons. Science 305, 187-188 (2004)

\section{Acknowledgments}

The authors thank Dr. S. Krishnamoorthy for providing silicon molds made using self-assembly. Useful discussion with Dr Ghim Wei Ho of the National University of Singapore is acknowledged. Jie Yong Chan is thanked for his assistance in experiments. The authors thank Mr. Sowmik for help in Raman measurements. One of the authors (BR) would like thank the A*STAR for supporting her stay in Singapore as a visiting scholar at IMRE. This work was supported by the IMRE-funded core project no. IMRE/09-1C0319. Support from Department of Science and Technology, India, is gratefully acknowledged.

\section{Author contributions}

B.R., G.U.K. and M.S.M.S. conceived the idea. B.R., S.H.L. and M.S.M.S. designed experiments. B.R. and S.H.L. performed experiments. B.R., S.H.L., M.S.M.S. and G.U.K. analyzed the data. B.R. and S.H.L. wrote the main manuscript text and prepared figures. All authors reviewed the manuscript.

\section{Additional information}

Supplementary information accompanies this paper at http://www.nature.com/ scientificreports

Competing financial interests: The authors declare no competing financial interests. License: This work is licensed under a Creative Commons Attribution-NonCommercial-NoDerivs 3.0 Unported License. To view a copy of this license, visit http://creativecommons.org/licenses/by-nc-nd/3.0/

How to cite this article: Radha, B., Lim, S.H., Saifullah, M.S.M. \& Kulkarni, G.U. Metal hierarchical patterning by direct nanoimprint lithography. Sci. Rep. 3, 1078; DOI:10.1038/ srep01078 (2013). 